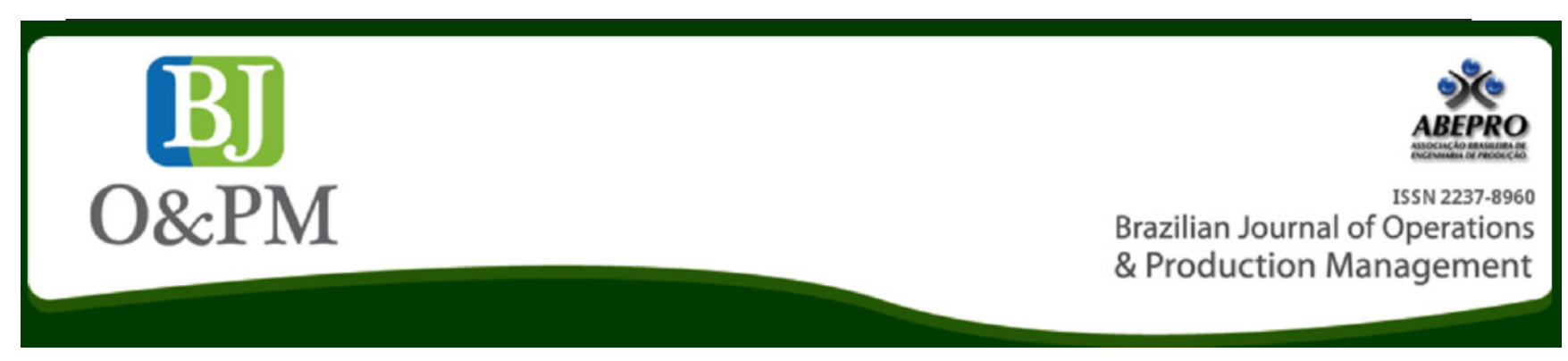

\title{
STORAGE MANAGEMENT OF CLINICAL RESEARCH SUPPLIES OF A PHASE IIB/III, NATIONAL, MULTI-CENTRE, DOUBLE-BLIND AND RANDOMIZED STUDY
}

Lila M. Haradaa; Enio Fernandes Rodriguesa; William de Paula Ferreiraa; Adriano Maniçoba da Silvaa; Luiz Teruo Kawamoto Júniora

${ }^{a}$ Federal Institute of Education, Science and Technology of Sao Paulo (IFSP) - Suzano, SP, Brazil

\begin{abstract}
New drugs suffer a long and slow proceeding to reach the market. On the one hand there are difficulties from the regulatory agency that delays the onset of clinical trial studies, on the other hand there is a slow patient recruitment during the course of the trial that prevents its completion. An efficient management of clinical trial supplies throughout the supply chain is essential. Thus, the objective of this study was to characterize the clinical supplies storage management in a national, multicentre, double-blind and randomized study to evaluate the efficiency and safety of a combination of drugs to treat acute pain. The study was primarily focused on storage conditions of clinical supplies, detailing their distribution to the research sites. According to the data observed in this study, the clinical supplies storage management was not determinant for the slow patient recruitment process that reflected the delay to the end of the study. And although the centers were located in different states, there were no delays, loss or damage during storage and distribution that could compromise the proper stock and storage management of clinical trial supplies.
\end{abstract}

Key words: Clinical Trial; Supply; Storage; Good practices. 


\section{INTRODUCTION}

Current economic reports have shown a great growth of the pharmaceutical market, both worldwide and in Brazil, pointing billions of dollars indexes in revenues, reaching trillions of dollars in the coming years (IFPMA, 2014, INTERFARMA, 2015). In this scenario, Brazil has emerged as the 6th largest pharmaceutical market in the world, growing the profitability of the pharmaceutical industry with commercialization of thousands of drugs (Brand name and generic drugs), all regulated by the official governing body of the country. On the other hand, regard the introduction of new drugs on the market, whether novel or innovative, there is a long and slow process by regulatory agency to approve the conduct of clinical studies to ensure safety and efficacy, and later, its commercialization. However, clinical studies are a very important and very expensive part of the drug development process, since it includes the production, distribution and administration of the test drug to volunteer patients from different geographic locations (Lee, 2006, Zucchetti et Morrone, 2012).

Since the pharmaceutical industry deals with an extensive drug portfolio to treat a broad range of diseases, a lot has been discussed about the complex supply chain management of drugs However, a little is said about storage management of newdrugs, those which are not yet commercially available but are used in clinical studies. With the production, the volume is intended specifically to a particular group of patients selected for a particular disease.

Unlike what happens with the storage of drugs for commercialization, which requires a complex strategic planning, the inventory of medical supplies that is stored for later distribution to research centers consists in a fixed, small and limited number testing drugs. The goal is that the stock be consumed as soon as possible, which signals the end of the clinical study stage. However, even handling small and limited quantities, storage of clinical supplies requires adequate conditions, inherent to medicines, besides additional confidentiality requirements, for being investigational products not commercially available yet. And it is due to the confidentiality that data on the progress of a clinical study are rarely disclosed.

The few existing scientific literature on clinical research supplies management direct to the problem caused by the globalization of these studies as a result of slow patient recruitment and high costs of clinical studies (Thiers et al., 2008). With the expansion of international multicenter studies, it is becoming increasingly difficult to have the right inventory in the right place at the right time and for the right patient. To optimize the supply plan and balance the various costs (manufacturing, storage, distribution, etc.) against the risk centers run out of the medication, or do not receive the necessary amount, some authors have shown planning approaches based on mathematical programming and simulations to improve the management of clinical supply chain, including demand forecasting (Abdelkafi et al.,2009, Fleischhacker et Zhao, 2011, Fleischhacker et al.,2015, Peterson et al., et al., 2004).

While it is evident that the bottlenecks of the regulatory agencies delay the onset of clinical research studies in Brazil, on the other hand, during the course of the trial, there is a slowdown in the recruitment of patients. It is imperative that the clinical supplies be available in the right quantities, at the right time in the right place, as recommended by Thiers et al., (2008). Thus, the study questions were: 1) How the storage management of supplies in a clinical study is performed, ensuring quality, integrity and confidentiality of testing drugs, following the official regulations of a research and development company of innovative medicines?; and 2) The way the storage management of clinical supplies are performed influences or disturbs the closure of the study and delay the application of its registration for the production and commercialization of investigational product along ANVISA?.

To answer these questions, this study was conducted in a research and development company of innovative medicines. And a clinical study of phase IIB/ III of an investigational product for the treatment of acute low back pain was taken as an example.

This study was divided into five sections, including this introduction. Section 2 presents theoretical framework, regards the pharmaceutical market, medical research, and Supply Chain Management of clinical trial. Section 3 presents the research methodology. Section 4 presents the results and discussion. Finally, section 5 presents the conclusions.

\section{LITERATURE REVIEW}

\subsection{Pharmaceutical Market}

The current scenario of the market has shown accelerated annual growth in sales of medicines, which has generated a high margin of profitability by pharmaceutical companies, both national and multinational.

According to the Association of Pharmaceutical Research (INTERFARMA) in the Brazilian pharmaceutical market in the last four years, 2010-2014, the pharmacy channel leveraged a profit of nearly $\mathrm{R} \$ 28$ billion to more than $\mathrm{R} \$ 40$ billion, corresponding to a commercialization growth of 76.3 billion to 121.6 billion doses of drugs. In the year 2015, it is predicted that the global pharmaceutical market reaches about \$ 1.1 trillion in profits, and the major emerging countries and generics will account for $28 \%$ of global sales with pharmaceuticals. Recent surveys by the IMS Institute for Healthcare Informatics shows only in the first quarter 
Brazilian Journal of Operations \& Production Management Volume 13, Número 4, 2016, pp. 430-441

DOI: 10.14488/BJOPM.2016.v13.n4.a3
2015 , drug sales rose $19.35 \%$, reaching a turnover of $R$ \$ 3.12 billion industry and sales nearly 200 million units (INTERFARMA, 2015). Predicting that in 2017, the global pharmaceutical market is expected to reach a $\$ 1.2$ trillion revenue (IFPMA, 2014).

In another pharmaceutical industry report, the Brazilian Association of Generic Drugs Industries (Pro Generics) analyzed market data between January 2014 and January 2015. From the 10 largest pharmaceutical companies operating in Brazil, generic drug sales accounted for 23, $7 \%$ of the revenues of these companies, corresponding to $\$ 6.2$ billion in sales, an amount $13.8 \%$ higher than the $\$$ 5.3 billion registered between January 2013 and January 2014. And among these 10 companies in the ranking, nine operating in the generics market and accounted for the production of 775 million units of medicine, the volume is $14.7 \%$ higher than the 661 million units in the same period last year (January 2013 to January 2014). In addition, in 2014 the generic industry also expanded its portfolio with the launch of 15 new products, which together add up $\mathrm{R} \$$ 1.6 billion in the year (ABRADILAN, 2015).

\subsection{Clinical research and studies of new drugs}

The development of drugs that may constitute effective and safe therapeutic alternative requires that the new substances under study are carefully tested and analyzed before they become available to doctors and patients. Conducting a clinical trial involves working in synergy between the pharmaceutical industry, the medical researcher, the group of patients, the ethics committee in research and regulatory agencies. It is this partnership, established with integrity and impartiality, which depends on the success of a clinical trial.

Clinical study or clinical research can be defined as any research conducted on humans in order to discover or confirm clinical / drug / or any other pharmacodynamics effects of the experimental drug/or identify any adverse reaction to the experimental drug / or to study absorption, distribution, metabolism and excretion of the investigational product to verify its safety and / or efficacy (ICH, 1996, ANVISA, 2015).

Clinical studies have different phases and can be classified as preclinical animal or human clinical phase to ensure the safety and effectiveness of a proposed treatment. In the preclinical stage, the new molecule with therapeutic potential is evaluated for toxic and pharmacologic effects through vitro and in laboratory animals. At this stage, only molecules with some pharmacological activity and a low toxicity profile are then tested on humans, which correspond to only $10 \%$ of substances initially developed (Lee, 2006, Lopes et Harrington, 2015).
In clinical phase, the phase I is to test the safety and dosage of the new active ingredient on a small group of volunteers (20-100 people), in healthy general, and pharmacokinetic and pharmacodynamics profile. In Phase II, they are carried over safely and efficacy testing on a larger group (100 to 200 persons), but these are already volunteers who have a particular disease or condition being treated. At this stage, one tries to get a better idea of how effective the treatment is and how safe the new drug is to establish the doseresponse relationship, the types of patients, the frequency of administration, or other safety features and therapeutic effectiveness. Depending on the study objectives, phase II can be subdivided into IIA and IIB. Phase IIA studies the effectiveness of the new drug or therapeutics scheme are evaluated, while in Phase IIB a promising treatment being tested and identified, in Phase III, studies (Lee, 2006, Lopes et Harrington, 2015).

In Phase III, assuming the success in Phase I and II it is confirmed the results of safety and efficacy on a larger group of patients, hundreds or thousands in several national and either international centers. At this stage, in addition, to determine the effectiveness and safety of the new drug, the most common adverse reactions, as well as the therapeutic profile and the demonstration of the potential advantages of the new therapy is compared to other already present in the market are identified. Assuming success in Phase III, the new drug is submitted to the official regulator of the country to obtain marketing approval. Phase IV is the observational phase of post-marketing surveillance, which is the studies after the drug is marketed to gather additional details about their effectiveness or safety profile (Lee, 2006, Lopes et Harrington, 2015).

According to information from the International Federation of Pharmaceutical Manufacturers \& Associations, PHRMA (2015), on research and development of medicines; the pharmaceutical research industry in 2014 invested approximately US $\$ 51.2$ billion in research and development of approximately 7,000 drugs around the world. It is estimated that its required between 10 to 15 years to develop a drug or a vaccine, and only one in 10,000 patients reaches compounds as a medicament test for their effectiveness and safety (INTERFARMA, 2015). Even though it is possible to know how many patients are needed at each stage of the clinical trial, is important to remember the existence of the inherent uncertainty associated with each test (the risk of study failure).

\subsection{Clinical research and regulation in Brazil}

All clinical studies should comply with the principles of Good Clinical Practice Manual of the International Conference on Harmonization, ICH (1996), and Good Clinical Practice (Documento-das-Américas, 2005). In 2009, ANVISA (National Health Surveillance Agency) published the 
Brazilian Journal of Operations \& Production Management

Volume 13, Número 4, 2016, pp. 430-441 DOI: 10.14488/BJOPM.2016.v13.n4.a3

Normative Instruction no.4 / 2009 establishing an inspection guide to verify compliance with Good Clinical Practice in clinical trials with medicines and health products in order to promote regulatory action in health surveillance, as well as ensuring the quality of results of effectiveness and safety obtained by all parties involved (ANVISA, 2009b). The rules for conducting clinical research in Brazil follow the resolutions of the National Health Council (CSN, 2013) and ANVISA (2008), which is the main drug industry organ in Brazil.

According to ANVISA surveys in 2011, Brazil's participation in the clinical studies phase I is quite insignificant (only $4 \%$ ), while the phase II reaches $22 \%$, and new drugs are registered as patents and tested for the first time on humans. Moreover, about $60 \%$ of clinical research activities are concentrated in the phase III studies, $11 \%$ in stage IV, but in this clinical research development universe in Brazil, $80 \%$ of the studies of new drugs are sponsored by multinational industries, indicating a low participation of national pharmaceutical companies (Lang, 2014, Molina, 2011, Zucchetti et Morrone, 2012). However, while Brazil stands as the world's seventh biggest economy and the sixth largest pharmaceutical market, it represents only $1 \%$ in participation of clinical studies in the world. It is mainly due the time spent on the approval of a clinical research, between 10 and 14 months in Brazil, while the average in the rest of the world is between four to six months. (Ferraz, 2009, INTERFARMA, 2015).

This is one of the major bottlenecks for the approval of clinical trials in Brazil, which passes through three fairly lengthy regulatory processes to get the ANVISA authorization for the clinical trial and can be conducted to prove the safety, effectiveness of the new drug, and the endorsement of the Ethics Committees of Research - CRE and the Ethics National Research Commission - CONEP (Nishioka, 2006).

One consequence of this slow pace of regulatory agencies is the lack of interest in emergence potential of many multinational pharmaceutical companies to include Brazil in its multicenter, where due to bureaucracy, and lack of recruitment terms of volunteer patients, who could guarantee even experimental access to treatment of diseases with innovative drugs which are not available yet . In a survey conducted by INTERFARMA in 2013 about the capability to conduct clinical studies in Brazil, based on the responses obtained from 18 member companies, it was found that the country lack the opportunity to perform 112 multicentric clinical studies, where 61 were submitted to ANVISA and they had no authorization in a timely manner, resulting in 3,712 patients who no longer benefit. The other 51 studies were lost before the estimated number of patients (INTERFARMA, 2015).

In addition, after completion of clinical trials proving its effectiveness and safety, so that a drug can be marketed throughout the national territory, the manufacturer shall submit to ANVISA the request for registration of that product. In this respect, Brazil also presents a great contrast compared to other countries. According to recent data from ANVISA, the analysis time (considering the waiting time in the analysis lists, time technical analysis, archiving time and the response time of the requirements for the regulated sector) can take an average of 997 days in the case of a generic, 850 days for a similar innovative, 631 days and 512 days for a new drug. In the rest of the world, these terms are between five months or a year (INTERFARMA, 2015).

\section{4 storage and management of clinical research supply chain}

Storage is the process of storing goods in an orderly and rational manner in a storage location specifically known as a warehouse, ensuring their proper conservation. Warehouses are products storage locations (raw materials, work in process, finished goods) with and between points of origin and points of consumption, usually temporary. Its main functions are to maintain the flow of materials throughout the supply chain to accommodate variability caused by factors such as: seasonality of products, batch production and transportation; consolidation of products from multiple vendors to deliver combined to customers; and processing as kitting, labeling and product customization (de Koster et al., 2007; Gu et al., 2007).

Storage and storage management are parts of a logistics management system, which is itself an essential component of any supply chain. Minimizing response time is a key goal, because it determines the processing power and system responsiveness. Today, it appears that companies are very concerned with the organization of goods within the warehouse in order to achieve high utilization of space and facilitate the efficient handling of the stored material. Competition in the market has required continuous improvement in design and operation of the distribution grids, which in turn requires higher performance warehouses. These requirements include real-time control of the warehouse operation, easy communication with other parts of the supply chain, and high levels of automation (Gu et al., 2007).

Although, storage management, inventory control and distribution of clinical supplies is one of the numerous procedures under the responsibility of a CRO (Contract Research Organization) or Representative Organization of Clinical Research, storage conditions of clinical research supplies must comply with the principles of Good Clinical Practice (ICH, 1996, Documento-das-Américas, 2005), guidelines of the Good Pharmaceutical Storage Practices (WHO, 2003) and Standard Operating Procedures specifications (POP) company. The clinic supplies should be stored in accordance with the protocol requirements and 
labeling. The location of the supplies must be identified and documentation of storage conditions should be maintained with the technician in charge, usually a pharmacist full time and aided by assistant technicians with training in pharmacy (Méthot et al., 2012).

At this time of a global economy, the clinical research studies are becoming larger and more complex, and therefore also the problems arise among many of the organizations involved, whether the customer, supplier or sponsor. All who are involved have a distinct common goal in reaching the start date of effective assessment and ensure that each patient for each research center is treated with the right medication at the right time. That is, a good supply management services can ensure high levels without retarding a clinical study because of the lack of medication in patients' recruiters centers, for example. Any delay in the completion of a clinical trial for a day means the loss of a day of exclusive patent and revenues associated with the sale of these drugs (Fleischhacker et al., 2015).

The clinical trial supply management is partly based on the standard supply chain concepts such as batch quantity, demand and eventual replacement. However, due to the specific constraints and the characterization of the variability inherent clinical supplies such as expiration date, availability of raw materials for the production of experimental medicine; development and implementation of experimental projects, recruitment and inclusion of patient dropouts, and distribution of test drugs are factors that generate significant uncertainty in the prediction of material needs, standard concepts cannot be applied in clinical research. This is because these global uncertainties in demand forecasting can lead to a risk to the clinical study when you cannot provide the right drug for the right patient at the right time (Abdelkafi e et al., 2009). In this case, due to increased uncertainty associated with the required excess is likely to increase significantly. These parameters must be carefully selected in conjunction with the determination of an optimal production plan to achieve the desired balance between cost and risk (Peterson et al., 2004).

In order to reduce this risk, various techniques can be used, such as increased loading rate, the rules for providing dynamic (e.g., transport to the place after randomization the patient), and common tracking in real time inventory. As each of these techniques involves a certain cost, the purpose of managing the supply of medical supplies is finding the right balance between these costs and risks for each attempt. Some authors have used simulationbased optimization methods that are considered effective and efficient alternative to solve a major problem of inventory management decision and demand clinical supplies (Abdelkafi et al., 2009, Fleischhacker et Zhao, 2011, Fleischhacker et al., 2015, Peterson et al., 2004).

The total cost of optimizing the clinical supply chain is still difficult to implement in practice, requiring a risk of translation cost despite its intangible aspect. Abdelkafi et al., (2009) showed in a clinical trial simulation approach with long-term supply of supply strategies that it is possible to optimize the supply plan and balance the various costs (i.e., manufacture, storage, distribution, etc.) against the risk of becoming centers without medication.

Among the contrast of an adequate and optimal management of clinical research supplies, and the reality that is in the chain production-storage-distribution, we can point out the most important factors that influence the process of conducting a clinical trial. It is important to remember that every trial is different from each other. In clinical supply chain, waste and unnecessary drug distribution may comprise a major part of the total cost of the study. Plan carefully, forecasting and clinical supply management can significantly reduce costs by ensuring that supplies reach the research sites at the right time.

The optimal supply management plan should strike a balance between the key factors to deliver medical supplies when and where needed at a minimal cost. The cost of clinical supplies is not only the manufacturing cost of medicines. It also includes the planning costs of supply chain management, purchasing, packaging, transportation and destruction (Schwemmin et Goldfarb, 2012).

Since the clinical trials supplies are available to start a clinical trial, a process of tracking and monitoring can ensure that clinical kits levels are maintained, and supply to each patient is continuous and uninterrupted, and an attention to exhalation the expiry date or availability of the comparator drug. On the other hand, when a clinical study is completed, it is necessary to consider the medication waste that remains in the research centers whose destruction also incurs costs, as grossly excessive production supply of drugs, testing is often considered a viable option in view of the risk of a lack of supplies which could lead to higher costs and substantial delays in closing the trial. For this system to work properly, a good storage management must be done from the clinical supplies which are produced and delivered to organizations responsible for monitoring the study.

\section{METHODS}

\subsection{Methodological Approach}

For this work, the methodology initially adopted was the literature in books, magazines and electronic documents, provides critical to the theoretical foundation necessary for discussion on the subject and, consequently, achieve the proposed objectives.

From the point of view of technical procedures, the design of this study was a case study because it involves the study that allows its broad and detailed knowledge within the investigated organization, but the results cannot be generalized to other companies (Yin, 2015). 
Brazilian Journal of Operations \& Production Management

Volume 13, Número 4, 2016, pp. 430-441

DOI: 10.14488/BJOPM.2016.v13.n4.a3

From the point of view of addressing the problem and the nature of the variables studied, the research can be classified as qualitative, where the process and its meaning are the main focuses of approach. The natural environment is the direct source for data collection and the researcher is the key instrument that has direct contact with the environment and the object of study in question, requiring more intensive field work (Prodanov et de Freitas, 2013).

\subsection{Case Study}

\subsubsection{Context of the companies involved}

Sponsor: National RD \& I company, a joint venture with equal capital of two large national pharmaceutical companies, to carry out research projects, development and innovation. The goal of the joint venture is to prospect new business and development of innovative medicines from its molecular creation, patent, pharmaceutical technology formulation, performing bioequivalence tests, stability, safety and efficacy, regulatory affairs to be approved by ANVISA for they can be produced and marketed by the respective investors' pharmaceutical industries. Developed the innovative drug.

Manufacturer of drug-tests: large national pharmaceutical industry, one of the shareholders of the joint venture. Produced pilot batch of drugs-testing ( $10 \%$ of the commercial lot).

CRO (Contract Research Organization) or Representative Organization of Clinical Research (CRO): small national company that managed and coordinated the clinical trial of the new drug in the research centers, as well as make storage management inventory of clinical research supplies and distribution centers as per the demand. Among other activities to conduct the clinical study, were also under the responsibility of the CRO assembly clinical kits, randomization, the review of the design, clinical protocol structuring, preparation of case report form (CRF Case Report Form) the adaptation of Informed Consent and Informed (IC) and patient information, quality control research, the selection of researchers and research centers and the training of these centers, submission of regulatory processes of the clinical study to CEP / CONEP / ANVISA, and monitoring of activities with the research centers.

Research center: to characterize the clinical study as multicenter, 6 researches centers were selected in several locations of the country, two in São Paulo (SP), one in Curitiba (PR), one in Porto Alegre (RS) one in Goiânia (GO) and one in Cuiabá (MT).

\subsection{Production of drug-testing (investigational product)}

For this work it was selected as an example the investigational product $\mathrm{XYZO23}$ to demonstrate how the storage management clinical supplies for the clinical study to evaluate the safety and effectiveness on humans. The XYZ023 is a combination of two drugs, a muscle relaxant action and other anti-inflammatory and analgesic to treat acute pain (up to 7 days duration). The mechanism of action proposed for this combination was that the XYZO23 promote synergism of action due to the different mechanisms for the relief of pain from muscle inflammation.

The investigational product was produced according to the standards of Good Practices of ANVISA (2010), in tablet form with dosages of $2.5 \mathrm{mg}$ and $5.0 \mathrm{mg}$ to evaluate the efficiency, which were compared with reference medicines. The production was done by the pharmaceutical shareholder industry as pilot batch (minimum amount that a drug must be produced to ensure that there is lot of homogeneity) and sought to play to maximize the technical, operational and manufacturing processes of the proposed industrial batch and ensure a high level of safety for the product and the process which are reproduced on an industrial scale. According to the regulation of ANVISA, the pharmaceutical company must make at least three batches of the medicine, each with a minimum amount equivalent to $10 \%$ of the planned industrial batch, or equivalent amount to the minimum capacity of industrial equipment to be used (ANVISA, 2009a).

The productions of pilot batches are essential for a more detailed assessment of the characteristics and quality of a product. With this production it is possible to carry out its assessment tests, the fundamental characteristics of a product before allowing their release for consumption, and enable the implementation of bio-pharmacokinetic tests when required. Thus, the production of these lots must play the maximum technical, operational and manufacturing processes of the industrial lot offered the product to be assessed for later clearance of its registration by ANVISA (ANVISA, 2009a).

The production of this pilot batch has been duly notified and filed with ANVISA. With the approval of the regulatory agency for meeting the required conditions, it was made available for use the manufacturer's criteria for clinical study with duly mentioning the product shelf life, counted starting from the date of effective manufacturing (ANVISA, 2009a).

\subsubsection{Installation of kits drug-testing for clinical research}

The clinical study XYZO23 with the investigational product has been termed as IIB / III phase, national multicenter, randomized, double-blind, comparative to evaluate the efficiency and safety of the drug combination for treating acute pain.

According to the clinical protocol, the study was defined to include 228 patients ( 57 per arm), to evaluate the efficacy and safety of drugs, testing for 7 days at a dose of two 
D Brazilian Journal of Operations \& Production Management Volume 13, Número 4, 2016, pp. 430-441

DOI: 10.14488/BJOPM.2016.v13.n4.a3 tablets per day. However, there were produced to stock an additional total of $20 \%$ as a reserve in the event of need for replacement due to loss or damage, if necessary.

They were assembled by sponsoring the four arms of the trial with 57 vials of each drug test containing 18 tablets, as follows:

- Drug test dose 1: 57 bottles;

- Drug test dose 2: 57 bottles;

- Reference Drug 1: 57 bottles;

- Reference Medicine 2: 57 bottles;

These tests drugs with respective bottles rescue medications were sent to the CRO for storage, labeling and randomization of clinical supplies kits and were subsequently distributed to research centers on demand, to be administered to patients recruited for the treatment of acute low back pain.

The objective randomization allocates equal numbers of participants in each treatment arm in order to optimize the power of the statistics to a fixed sample size. The randomization list was generated by the company responsible for the statistical analysis of the study using a validated system that automatically designates the randomization of the treatment groups to randomization numbers in the proportion specified for 228 patients, according to clinical protocol.

Thus, the 228 elective kits clinical research (57 per arm) were randomized by $\mathrm{CRO}$ and followed the statistical criteria to be double-blind, meaning neither the investigator (physician) or the patient would know which drug would be taking, as follows :

- 01 drug test dose bottle $1+01$ medicine bottle redemption OR;

- 01 drug test bottle dose $2+01$ medicine bottle redemption $\mathrm{OR}$;

- 01 drug bottle Reference 1 + 01 medicine bottle redemption $\mathrm{OR}$;

- 01 drug bottle Reference $2+01$ medicine bottle rescue.

The drug used in this study was to rescue Paracetamol, an anti-inflammatory and analgesic amount of 14 tablets per bottle, which were added to the medical kit to ensure that if the drug test did not effect, the patient could take to ease the pain.

Each vial of drug test and drug rescue, properly identified as such, was placed in a box of adequate proportions, and then stored the pharmacy premises CRO, according to (WHO, 2003).

\subsubsection{Data collection and analysis}

Data on production, storage and distribution of pilot batch of medicines and installation of clinical research kits were collected from the manufacturer / sponsor. Since the data on the storage management, inventory, and distribution of medical kits to research centers they were obtained from CRO that managed the clinical trial.

The results were qualitatively analyzed and presented in graphs, showing the profile of the management storage drug kits in CRO and sent to the six centers participating in the clinical study during the 12 month period and the demand profile for each study.

\section{RESULTS AND DISCUSSION}

The logistics of dealing with the effective storage, distribution and regulatory compliance can be complex and complicated in the case of clinical research supplies. However, if you follow the established best practices, it can ultimately help in simplifying new drug development trajectory through the clinical trial process. The scenario in which a team operates clinical research should be organized in order to increase its efficiency to conduct a clinical trial in accordance with Good Clinical Practice (ICH, 1996, Documento-das-Américas, 2005).

In this work it was evaluated and made for the management of stored medical supplies initially in CRO and later distributed to educational centers during the first 12 months of the study, which was the period of validity remaining from the first batch of medical kits, and identify which influence factors or difficult the termination of the study at the six centers participants clinical study.

According to the clinical protocol, this study should include a total of 228 patients to test the product investigational XYZO23 for treatment of acute low back pain in a double blind study randomized. 228 vials containing the four arms of testing drugs and their rescue medications were sent to the CRO who managed this clinical study, following the Standard Operating Procedures protocols (POP) of the company and applicable local laws.

The procedures and clinical supply storage features are similar in all studies, but the way they are made and carried out can make a huge difference in the development of clinical research, resulting from good storage management and chain of clinical supplies.

After receiving the study by Sponsor medication, an inventory of product research was made by $\mathrm{CRO}$, where the shipping instructions were reviewed to confirm that they have followed all requirements of the sponsor to ensure that the information such as, the number and the amount of the shipment match the incoming and outgoing products, as 
Brazilian Journal of Operations \& Production Management

Volume 13, Número 4, 2016, pp. 430-441

DOI: 10.14488/BJOPM.2016.v13.n4.a3

well as checking the integrity of the investigational product. All documentation related to shipping and receiving experimental drugs were maintained as an essential document of the study.

After identifying as the sponsor, supplies were accounted for labeled, randomized and double-blind. Randomization was made so that each participant had the same chance of receiving any of the interventions, and the assignment was performed using a mechanism so that neither the participant nor the researcher could know in advance what are the treatments which patients would submitted (Suresh, 2011).

The labeling of bottles were made by the responsible technician and revised by his assistants to ensure that each arm of the study was correctly identified. Labels contain the product code, expiry date, storage conditions and batch number, as well as the name and address of the sponsor / investigator and protocol identification. The label also specifies any other special precautions for the product.

When installing the clinical kits, test medication vials and rescue medication were placed in a properly identified box, which would then be sent to the study centers. The storage location within the CRO premises had four different rooms: one for delivery of supplies; another for labeling and accounting; the third for storage proper clinical supply agreement, with each sponsor for exclusive use of cabinets for this clinical study, properly locked and under controlled temperature ( $15 \circ \mathrm{C}$ to $25 \circ \mathrm{C}$ ) with humidity (between 40 and $70 \%)$; and a room for the storage of returned goods to be destroyed by the sponsor after accounting and reconciliation thereof. The Access to each of the rooms was kept under lock for safety and monitored by cameras to avoid the traffic of unauthorized persons. Any clinical supply was stored properly according to the recommendations of the Guide to Good Practice for Pharmaceutical Products Storage of and POP company (WHO, 2003).

Throughout the process of the clinical study, it is important to maintain records associated with the receipt, storage and dispensing of a product under investigation. It is known as providing drug accounts of the study. While the study sponsor is responsible with production of the investigational product, packaging and distribution of the product for the study sites, it is the center's responsibility to maintain proper records of handling and dispensing products. (SCHWEMMIN et GOLDFARB, 2011) During the course of the study, the following information was properly recorded for accounting of medication used: the patient's identification code for which the medication was delivered, the date and the amount of dispensed medication and date and the amount of medication by the patient returned. These records were checked for the patient compliance and medication accounting form which was available for inspection by the study monitor and government health agencies.

Clinical supplies kits were sent to study centers in suitable containers by $\mathrm{CRO}$, along with an invoice itemizing the amount of drug contained in each delivery to assist the study team to maintain adequate and accurate records regarding the receipt, dispensing and return study medication. The transit time of CRO deposits kits for research center were the order of one day to the city of São Paulo, and two days for the other cities.

CRO kept storage of clinical supplies throughout the year on the premises and the initial shipment of each of the five centers were initialized kits necessarily following the randomization sequence. As it can be seen in Figure 1 , as the kits were consumed, sending new kits resulted from the application of the centers, in varying numbers shipment. Depending on the application, the CRO sent 3-7 kits per month for each center, so that they could maintain an average stock of five clinical supplies to meet demand. But even after 12 months of initiation, the study has not been completed, since in this period were included only $79 \%$ of patients of a planned total of 228 in the clinical study protocol in the six participating centers.

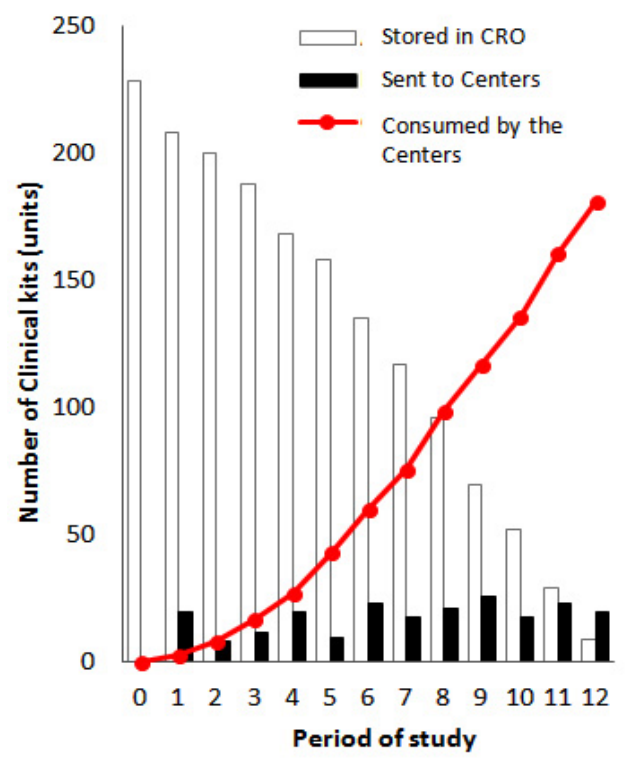

Figure 1: Profile of drug kits management stored in the ORC and sent to the 6 centers participating in the clinical study

Source: Author(s) own

It can also be seen in Figure 1 that in the first months after the start of the study itself, the demand for starter was quite low, but over the months, there has been a growing evolution of demand, but not at the speed expected by the sponsor who wanted to quit study in 6 months or within the period of validity of the medicine kit. However, it should be noted early during the first 4 months it was the participation of only 4 study centers; two centers were subsequently 
contacted to be part of the multicenter study. This may justify the low initial demand of the kits by the centers, whereas an increase in demand was most clearly evidenced when the six centers were active.

Confirming the above observations, in Figure 2 there is such a sharp change in the rate of recruitment of patients in each center even with the demand supplies have been adequately supplied with providing kits for the CRO without delay, loss or damage to maintain a regular inventory level in all centers. Assessing the regional profile, as centers of Sao Paulo demanded more receipts kits to promote more inclusion in the study, the centers of the south (Porto Alegre and Curitiba) had poor performance. The centers of the Midwest began operating four months after the other centers achieved the average demand. This suggests that perhaps there had been an influence of the location of research centers to a greater or lesser demand for clinical kits, or the efficiency of research centers to recruit patients, as the profile of patients who should be selected for the use of the investigational product corresponded a very common pathology. But even with an efficient service of research centers, it did not ensure that the recruitment of patients was also efficient.

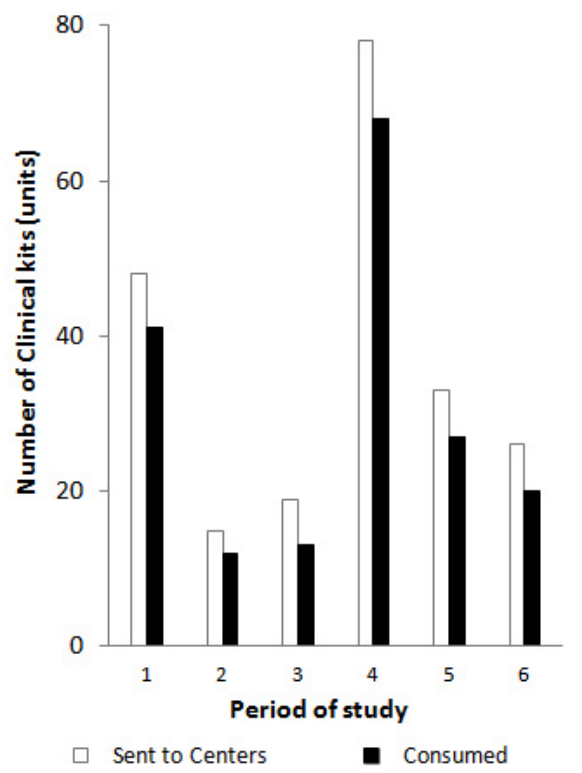

Figure 2: Profile of demand evolution of medical kits in the study centers. Centers 1 and 4 (Sao Paulo); Center 2 (Porto Alegre); Center 3 (Curitiba); Center 5 (Goiania); and Centre 6 (Cuiabá).

Source: Author(s) own

The recruitment of patients is a typical bottleneck in the clinical trials, and $80 \%$ of clinical trials fail to meet their patient recruitment periods (Getz and De Bruin, 2000). Other indirect causes may also have influenced the diversity of this demand. One of them may be the low adherence to study because during the treatment period, patients are required to follow the pre-defined business profiles, which also determine the drug dispensing agendas. However, some patients may drop during the course of treatment for various reasons, such as loss of interest due to dissatisfaction no observed improvement improves the processing time and see relieved of the symptoms of the disease or changes in personal life.

Figure 3 summarizes the timeline for completion of the clinical phase of the product XYZO23.

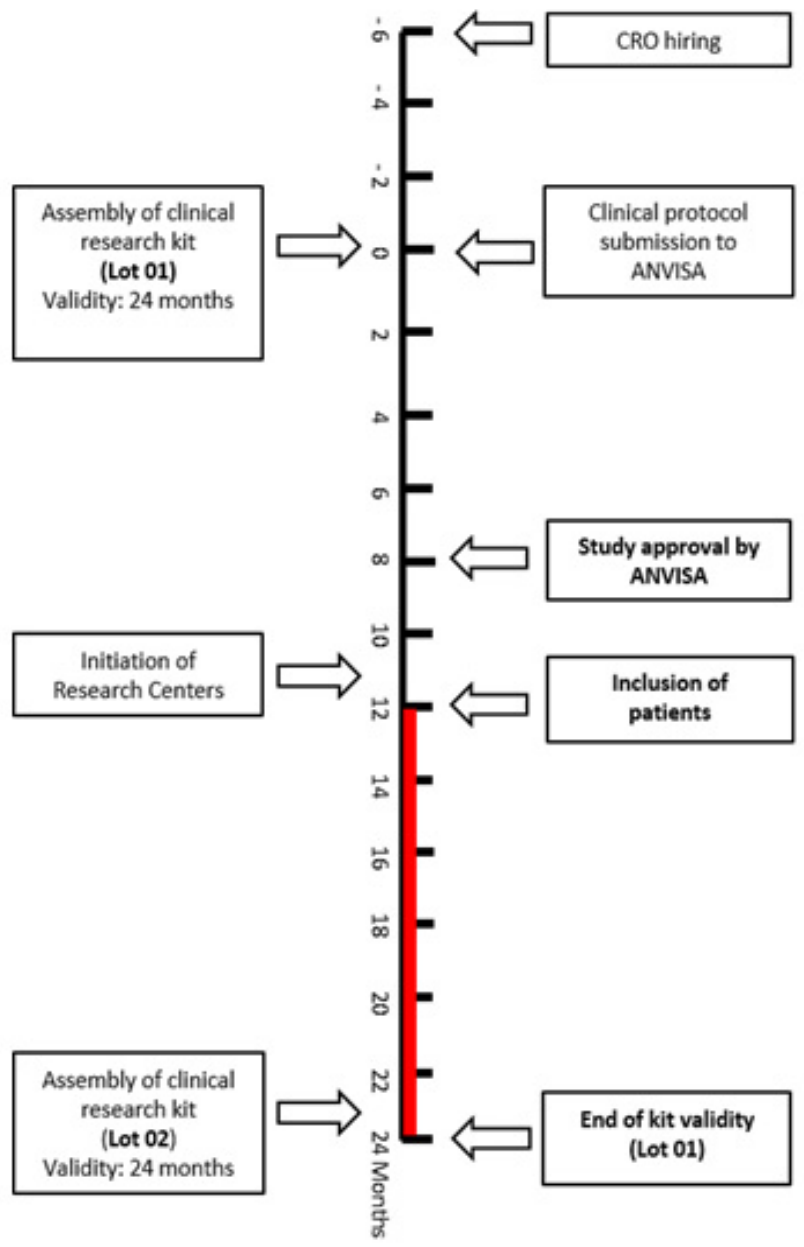

Figure 3: summarizes the timeline for completion of the clinical phase of the investigational product XYZO23.

Source: Author(s) own

As it can be seen the clinical procedure of study began approximately 14 months before the start of patient inclusions. The process began with the hiring of a CRO who was responsible for the study of management regarding the clinical, regulatory and the chain of clinical supplies. The CROs and research centers play a big role, whether a trial will be held on time and within the planned budget sponsor. This phase is also critical because it takes place in the preparation and submission it to ANVISA the clinical protocol of the drug test to obtain authorization to start the study with patients in selected research centers. This waiting latency period lasted for 8 months in this study. On this occasion were produced lots of test medication and comparators, 
Brazilian Journal of Operations \& Production Management

Volume 13, Número 4, 2016, pp. 430-441 DOI: 10.14488/BJOPM.2016.v13.n4.a3

and the mounting of clinical kits and thus begin the counting period of validity of the medication. Generally, batches are produced when submitting the application for authorization to ANVISA, because if the protocol received approval, the studies could be initiated, but these must be pre-tested for quality and product stability. During this period the research centers in different cities were also defined to characterize the study as multicentric. When the study was approved by ANVISA, the next two months were consumed to formalize the study in the centers and only then, the study can be officially launched.

One of the major differences in the storage management is when it comes to the area of clinical research, it is the amount of stock to be handled a fixed, small and limited number without replacements. The drug tests for a clinical study generally ranging from 200 to 2,000 units. The number varies according to the type of study and the complexity of the pathology to be investigated where in international multicenter; this number could reach 4,000 units, indicating that this is the number of patients to be recruited for the study. Another feature of the clinical supplies is only the production of a pilot lot which must meet the demand for the development of clinical stage, except when there is loss of expires and a new batch is arranged for replacement and continuation of the study. The term of validity also has an impact on the planning and supply management. In many cases, medical supplies should be made prior to the availability of long-term stability data.

In this study, as the duration of treatment was just a week, it was expected that clinical stage could be completed in a few months, before the batch validity of the end; however, this was not possible due to various factors involved driving clinical studies, such as the difficulty of recruiting patients. The drugs with expired validity were collected study centers for accounting, reconciliation and destruction by the sponsor, and replaced properly following the order of randomization. Generally, every batch that produced drugs have a period of maximum validity of 24 months, as in the case of the tests and comparators used in this study drugs, but due to the slowness of the regulatory process, the batch expiration time has been drastically reduced to 12 months study.

Another important aspect of medical supplies in the storage management refers to the costs of this over procedure of the clinical study. In traditional storage, costs reflect the amount of product stored on site that is in constant movement. The cost is based on factors such as volume of goods to be handled and stored, the time that a particular warehouse space will be occupied, the number of separate items in the combination product, which either need special requirements or restrictions for storage, the average size of request due out and the amount of administrative work required, including maintaining inventory records and preparation of bill of lading (de Koster et al., 2007). In the clinical approach, cost means a predefined amount of supplies that will be sent to each of the participating centers, with a fixed expense for that storage for the duration of the study. Proportionally, clinical supplies occupy volume, weight and very small amount.

In the case of supplies of this study the clinical starter 288 only occupied a volume of $0.23 \mathrm{~m} 2$ within a locked cabinet in a room with appropriate storage conditions and safety. However, the storage cost of the supplies remained constant throughout the study under contract so, because besides storing function, CRO also instructed its packaging, accounting, distribution to research centers, and accompanying destruction the study products in accordance with applicable local laws. However, this cost is less than $1 \%$ of total spending on the development of the entire clinical phase to test a drug. According to HOVDE (2006), expense with CROs is the third area of higher cost in spending on clinical research. Most of the expenditure on CRO is done by contracted service monitoring, site management and project, although other activities such as central laboratories can be outsourced. The total market for CROs and central laboratories is about $\$ 4$ billion, and the central laboratories are responsible for 800 million dollars.

Annual spending on drug delivery chain in clinical trials can be substantial, accounting for $20 \%$ or more of the expenses of a company in research and development of only one drug candidate, namely that a company can spend millions of dollars each quarter to produce only materials for a clinical trial. When failure in a clinical trial occurs every dollar spent on manufacture, storage and dispensing of unused clinical trials Clinical Supplies are wasted and, in most cases, the unused material should be returned to a proper disposal site for destruction (Fleischhacker et Zhao, 2011).

\section{CONCLUSION}

Clinical studies alone are very expensive, even when the pharmaceutical industry glimpses the enormous profitability of new drug. However, we come across that these studies that tend to have a very slow process until its registration approval by official regulatory agencies.

In Brazil, the major bottleneck of conducting clinical research is the long delay to obtain the approval of the study by ANVISA (INTERFARMA, 2015). The first stage of this bottleneck is to obtain approval for these studies be tested in selected centers. In this study, there were 8 months of waiting since the submission of authorization request, so that it could be started. However, beyond the slowness of an obstacle in the regulatory process at ANVISA, after the start of the study, also occurs frequently slow in the inclusion of patients who have the desired profile according to the criteria established by the sponsor, and who agree 
to participate in all stages of the study. As faster occur the safety assessment and efficiency of the new drug trial in humans, faster will be its introduction in the market, and the clinical demand of supplies is the speed flag that indicates when this step is complete. In this study, it was observed that the CRO properly played their role in managing the storage of clinical supplies in order to maintain their integrity and provide their distribution to the various study centers according to demand. However, regards the slow progress in the study, which was unable to complete the clinical phase in 12 months, one of the factors found was the difficulty of recruiting patients, often due the lack of adherence to protocol. On the other side, although the study centers were located in different states, there was no delay, loss or damage to the distribution of supplies that would compromise the proper management of the stock.

Any delay in this phase of the study leads to delay in completion, meaning a day less patent protection and revenues associated with the marketing of medicine. However, is important to note that this study showed the storage management of only one investigational product in a clinical trial, and with a very limited number of medical supplies monitored. We must remember that the CRO manage several dozen clinical study concurrently from different sponsors, which have several drugs for testing and, depending on the type of study, the number of patients to be recruited can reach thousands. Each study has its own characteristics and complexity in the ongoing clinical phase, but some procedures such as storage management of clinical supplies are similar across studies.

In recent years, it has been observed that great extent international multi-center clinical studies that seek the inclusion of thousands of patients in several countries to test new drug, are transferring the responsibility for management of clinical research supplies to large logistics pharmaceutical networks. This new membership to specialized logistics companies in confidential investigational products, results in an attempt to reduce hidden costs in the process of clinical research with storage activities, distribution, inventory and collection of drug from research centers.

\section{REFERENCES}

ABDELKAFI, C., BECK, B. H., DAVID, B., DRUCK, C.; $\mathrm{HOROHO}, \mathrm{M}$. 2009. Balancing risk and costs to optimize the clinical supply chain-a step beyond simulation. Journal of Pharmaceutical Innovation, 4, 96-106.

ABRADILAN. 2015. Associação Brasileira dos Distribuidores de Laboratórios Nacionais. Genéricos somam 23,7\% do faturamento das 10 maiores indústrias farmacêuticas. Available: Available at: http://www.abradilan.com.br/index. php?m=noticiaFE\&id_noticia=1326. Accessed 02 Jun 2015.
ANVISA. 2008. Agência Nacional de Vigilância Sanitária. Resolução - RDC No- 39, de 5 de junho de 2008. Aprova o regulamento para a realização de pesquisa clínica e dá outras providências. Publicado no D.O.U. n.107, em 6 Jn 2008.

ANVISA. 2009a. Agência Nacional de Vigilância Sanitária. Instrução Normativa n.2, de 30 de março de 2009. Determinar a publicação do Guia para Notificação de LotesPiloto de Medicamentos. Publicado no D.O.U. n.62, seção 1, p.1 em 01 de abril 2009a.

ANVISA. 2009b. Agência Nacional de Vigilância Sanitária. Instrução Normativa no 4, de 11 de maio de 2009. Dispõe sobre o Guia de Inspeção em Boas Práticas Clínicas. Publicado no D.O.U. em 12 de maio de 2009b.

ANVISA. 2010. Agência Nacional de Vigilância Sanitária. Resolução - RDC no 17, de 16 de abril de 2010. Dispõe sobre as Boas Práticas de Fabricação de Medicamentos. Publicado no D.O.U. em 19 de abril de 2010.

ANVISA. 2015. Agência Nacional de Vigilância Sanitária. Resolução - RDC $n^{\circ}$ 9, de 20 de fevereiro de 2015. Dispõe sobre o Regulamento para a realização de ensaios clínicos com medicamentos no Brasil. Publicado no D.O.U. em 03 de março de 2015.

CSN. 2013. Conselho Nacional de Saúde. Resolução CNS no. 466, de 12 de dezembro de 2012. Aprova as diretrizes e normas regulamentadoras de pesquisas envolvendo seres humanos. Publicada no D.O.U. de 13 de junho de 2013.

DE KOSTER, R., LE-DUC, T.; ROODBERGEN, K. J. 2007. Design and control of warehouse order picking: A literature review. European Journal of Operational Research, 182, 481501.

DOCUMENTO-DAS-AMÉRICAS. 2005. Boas Práticas Clínicas. IV Conferência Pan-Americana para Harmonização da Regulamentação Farmacêutica. República Dominicana 2-4 de Março de 2005.

FERRAZ, O. L. M. 2009. Análise Crítica do Sistema CEP/ CONEP e Estudo Comparativo Internacional de Aprovação Ético-Regulatória em Pesquisa Clínica - Sugestões para - Aperfeiçoamento do Sistema CEP/CONEP no Brasil. Sociedade Brasileira de Medicina Farmacêutica. Available: Available at: Análise Crítica do Sistema CEP/CONEP e Estudo Comparativo Internacional de Aprovação Ético-Regulatória em Pesquisa Clínica - Sugestões para o Aperfeiçoamento do Sistema CEP/CONEP no Brasil. Sociedade Brasileira de Medicina Farmacêutica. Accessed: 06 Jun 2015.

FLEISCHHACKER, A., NINH, A.; ZHAO, Y. 2015. Positioning inventory in clinical trial supply chains. Production and Operations Management, 24, 991-1011.

FLEISCHHACKER, A. J. \& ZHAO, Y. 2011. Planning for demand failure: A dynamic lot size model for clinical trial 
supply chains. European Journal of Operational Research, 211, 496-506.

GETZ, K. A.; DE BRUIN, A. 2000. Breaking the development speed barrier: Assessing successful practices of the fastest drug development companies. Drug information journal, 34, 725-736.

GU, J., GOETSCHALCKX, M.; MCGINNIS, L. F. 2007. Research on warehouse operation: A comprehensive review. European journal of operational research, 177, 1-21.

HOVDE, M. 2006. Management of clinical development costs. In: Lee, C.H.. Lee, L.H.; Wu. C.L; Lee, B.R.; Chen, M.L., ed. Clinical Trials of Drugs and Biopharmaceuticals. Boca Raton: CRC Press, 2006. p. 87-102.

ICH. 1996. International Conference on Harmonisation. Guideline for Good Clinical Practice, E6(R1). Available: Available at: http://www.ich.org/fileadmin/Public_ Web_Site/ICH_Products/Guidelines/Efficacy/E6/E6_R1_ Guideline.pdf. Accessed: 06 Jun 2015.

IFPMA. 2014. International Federation of Pharmaceutical Manufacturers \& Associations. The pharmaceutical industry and global health. Facts and figures. Available: Available at: http://www.ifpma.org/fileadmin/content/ Publication/2014/IFPMA_-_Facts_And_Figures_2014.pdf Accessed: 06 Jun 2015.

INTERFARMA. 2015. Associação da Indústria Farmacêutica de Pesquisa. Comparações Internacionais no Comércio de Produtos Farmacêuticos e Balança Comercial Brasileira de Medicamentos. Evolução em 10 anos. São Paulo: Interdoc.

LANG, D. 2014. Brasil perde oportunidades no setor de pesquisa clínica. ABRACRO. Available: Available at: http:// www.abracro.org.br/informacoes-utilidades/noticias/168brasil-perde-oportunidades-no-setor-de-pesquisa-clinica Accessed: 06 de Junho de 2015.

LEE, L. H. W., C.L.; LEE, B.R.; LEE, C. J. 2006. Clinical Trials from Phase I to Phase IV. Boca Raton: CRC Press, p.113-120.

LOPES, R. D.; HARRINGTON, R. A. 2015. Compreendendo a Pesquisa Clínica, AMGH Editora.

MÉTHOT, J., BRISSON, D.; GAUDET, D. 2012. On-site management of investigational products and drug delivery systems in conformity with Good Clinical Practices (GCPs). Clinical Trials, 9, 265-271.

MOLINA, D. 2011. Anvisa divulga perfil de pesquisa clínica de medicamentos no Brasil. Anvisa.

NISHIOKA, S. A. S., P.F.G. 2006. A Agência Nacional de Vigilância Sanitária e a pesquisa clínica no Brasil. Rev Assoc Med Bras, 52, 60-2.

PETERSON, M., BYROM, B., DOWLMAN, N.; MCENTEGART, D. 2004. Optimizing clinical trial supply requirements: simulation of computer-controlled supply chain management. Clinical Trials, 1, 399-412.

PHRMA. 2015. Pharmaceutical Research and Manufacturers of America. 2015 Biopharmaceutical Research Industry Profile. Washington, DC: PhRMA.

PRODANOV, C. C.; DE FREITAS, E. C. 2013. Metodologia do Trabalho Científico: Métodos e Técnicas da Pesquisa e do Trabalho Acadêmico-2 a Edição, Editora Feevale.

SCHWEMMIN, R.; GOLDFARB, N. M. 2012. Clinical Trial Supply Fundamentals.

SURESH, K. 2011. An overview of randomization techniques: an unbiased assessment of outcome in clinical research. Journal of human reproductive sciences, 4, 8.

THIERS, F. A., SINSKEY, A. J.; BERNDT, E. R. 2008. Trends in the globalization of clinical trials. Nature Reviews Drug Discovery, 7, 13-14.

WHO. 2003. Guide to good storage practices for pharmaceuticals. World Health OrganizationTechnical Report Series, No. 908, Annex 9, 2003.

YIN, R. K. 2015. Estudo de Caso-: Planejamento e Métodos, Bookman editora.

ZUCCHETTI, C.; MORRONE, F. B. 2012. Perfil da pesquisa clínica no Brasil Profile of clinical research in Brazil. Revista HCPA, 32, 340-347. 